based specialist in orthodontics and dento-facial orthopedics. The author aims to illustrate the treatment philosophies of world-renowned specialists in prosthodontics, periodontics, orthodontics, implantology, dental technology, maxillofacial surgery and restorative dentistry through a series of interdisciplinary treated cases.

The book compromises 28 chapters and is beautifully presented and reader friendly. It is made up of four sections. The first covers patient evaluation and diagnosis in relation to multidisciplinary treated cases with high aesthetic needs and highlights the importance of understanding the management of occlusion when treatment planning.

Interdisciplinary treatment planning of implant cases including complex cases, treatment of an implant failure and an innovative approach to guided bone regeneration forms the majority of the second section.

The third section covers the minimally invasive approach to restorative dentistry .A series of differing restorative cases are used to illustrate important aesthetic and restorative dental procedures used in common clinical situations.

A range of patient-centred cases is presented in the final section, which emphasises the need to tailor treatment to the individual patients' needs.

In each chapter the case treated is discussed in relation to the philosophical basis of the clinician for the treatment provided, why that particular case was chosen and any special challenges met by the clinician. In addition, a retrospective evaluation of the case is provided whereby the treatment is appraised and assessed as to how it could have been improved upon.

The quality of illustrations is excellent. The patient records presented include numerous before and after, life-like colour photographs that comprehensively show the stepwise stages of the clinical treatment and in certain cases the accompanying technical and laboratory procedures. This gives the reader a clear understanding and is complimented by the accompanying text, which is concise yet fully explains the treatment stages.
This book provides a philosophy of excellence based upon sound interdisciplinary assessment, diagnosis and treatment planning for many everyday clinical situations, producing optimal results.

Overall this is a substantial, very well illustrated and very readable book. It provides key learning in the theoretical and practical multidisciplinary approach to assessment, diagnosis and treatment planning of simple and complex cases experienced by the specialist and general dental practitioner. The author manages to navigate the reader through a complex subject in a straightforward and approachable way.

M. O'Donovan

\section{ITI TREATMENT GUIDE VOLUME 6: EXTENDED EDENTULOUS SPACES IN THE ESTHETIC ZONE}

\begin{tabular}{|c|c|}
\hline $\begin{array}{l}\text { ITI } \\
\text { Treatment } \\
\text { Guide }\end{array}$ & $\begin{array}{l}\text { J. G. Wittneben, H. P. Weber } \\
\text { Quintessence } \\
\text { price } £ 68.00 \text {; pp } 276 \\
\text { ISBN } 9783868671414\end{array}$ \\
\hline 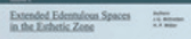 & \\
\hline Q & \\
\hline
\end{tabular}

An extended edentulous space in the aesthetic zone is described by many as the most challenging situation in implant dentistry. Written by a team of experts, this text aims to provide clinicians with practice orientated and evidence-based information on procedures for placing and restoring multiple implants in such situations.

The text is broken up into nine well laid-out chapters. It begins with a review of the available evidence upon which the text is based. Usefully, it sounds words of caution for areas where the current literature is lacking.

It moves on to an excellent chapter on pre-operative evaluation and treatment planning. Risk assessment is an important factor for the planning of any case and here the chapter excels by providing several easy to follow tables that act as a quick reference guide for classifying case complexity.

Surgical considerations are discussed in depth. Cone-beam CT images back up the recommended prosthodonticallydriven approach to surgical planning. There is plenty of information on timing of implant placement after extractions, degree of ridge resorption, the conditions required for a staged or simultaneous bone augmentation approach, and number and location of implants required for different cases.

A chapter on prosthodontic considerations follows. Each step from provisionalisation to final restoration is explained in detail. The use of screw or cement-retained implants is compared, along with a description of factors to be considered in abutment selection.

Chapter six is the highlight of this text. Nine clinical cases are presented and described in fascinating detail. They cover everything from the initial complaint, to treatment planning, to procedural techniques. There are exceptional photographs to describe each stage and all cases vary, covering a diverse range of situations.

The text ends with a useful chapter on causes, prevention and management of complications, with real-life examples providing the realisation that this treatment option is not without risk.

In summary, I found this text very educational. The easy to follow layout eases your understanding of the various steps involved in formulating a complex treatment plan, whilst also providing plenty of useful information that can be taken and used in any case. Everything is backed up with highquality images and descriptions of real-life examples. I would highly recommend the text to anyone with a keen interest in implants. It assumes a good understanding of implantology already, but I would not let this dissuade the novice from reading it.

E. Nugent 